“ (C) 2017 IEEE. Personal use of this material is permitted. Permission from IEEE must be obtained for all other uses, in any current or future media, including

reprinting/republishing this material for advertising or promotional purposes, creating new collective works, for resale or redistribution to servers or lists, or reuse of any copyrighted component of this work in other works." 


\title{
A Wireless Steady State Visually Evoked Potential-based BCI Eating Assistive System
}

\author{
Ching-Yu Chiu ${ }^{1}$, Avinash K Singh ${ }^{1,2}$, Yu-Kai Wang ${ }^{1,2}$, Jung-Tai King ${ }^{2}$, Chin-Teng Lin ${ }^{1,2}$ \\ ${ }^{1}$ Faculty of Engineering and Information Technology, University of Technology, Sydney, Australia \\ ${ }^{2}$ National Chiao Tung University, Hsinchu, Taiwan
}

\begin{abstract}
Brain-Computer interface (BCI) which aims at enabling users to perform tasks through their brain waves has been a feasible and worth developing solution for growing demand of healthcare. Current proposed BCI systems are often with lower applicability and do not provide much help for reducing burdens of users because of the time-consuming preparation required by adopted wet sensors and the shortage of provided interactive functions. Here, by integrating a state visually evoked potential (SSVEP)-based BCI system and a robotic eating assistive system, we propose a non-invasive wireless steady state visually evoked potential (SSVEP)-based BCI eating assistive system that enables users with physical disabilities to have meals independently. The analysis compared different methods of classification and indicated the best method. The applicability of the integrated eating assistive system was tested by an Amyotrophic Lateral Sclerosis (ALS) patient, and a questionnaire reply and some suggestion are provided. Fifteen healthy subjects engaged the experiment, and an average accuracy of $91.35 \%$, and information transfer rate (ITR) of $\mathbf{2 0 . 6 9}$ bit per min are achieved. For online performance evaluation, the ALS patient gave basic affirmation and provided suggestions for further improvement. In summary, we proposed a usable SSVEP-based BCI system enabling users to have meals independently. With additional adjustment of movement design of the robotic arm and classification algorithm, the system may offer users with physical disabilities a new way to take care of themselves.
\end{abstract}

Keywords - Steady state visually evoked potential (SSVEP); BCI; Robotic arm; Eating assistive system

\section{INTRODUCTION}

The demand of healthcare has been growing due to the facts that population ageing is happening in all regions and there are currently no biomedical treatments available that can reverse the deterioration of motor function caused by ageing or other neurological conditions, such as spinal cord injury (SCI), traumatic brain injury (TBI), and stroke [1]. At the same time, under general healthcare situations, the physiological and psychological burdens are heavy for both caregivers obligated to take care of someone anytime and anywhere and care receivers not having the ability to take care of themselves. Therefore brain-computer interfaces (BCIs) which aims at enabling users to perform tasks through their brain waves has been a feasible and worth developing solution to this plight [24].

BCIs achieve their objectives through procedures which consist of signal acquisition, signal processing, and applications [5]. There are lots of methods for these three aspects of BCIs which have been proposed and each has its own pros and cons. Therefore, how to integrate these methods effectively is another topic worthy of concern.
Two key issues related to signal acquisition are target brain patterns and data transmission. The most common used brain patterns of BCIs are P300 potentials, steady-state visual evoked potentials (SSVEP), and motor imagery $[6,7]$. SSVEP provides high signal-to-noise ratio (SNR), high information transfer rate (ITR) and minimal user training and thus is widely adopted [8]. On the other hand, data transmission of BCIs can be classified as wired or wireless. Wired BCI systems are generally with better signal quality but also bulky and heavy; more importantly, they often limit the users' movement in ordinary working or living environments. Such systems are thus impractical for reallife use. Therefore, for a BCI system to be more applicable, it needs to be wireless but not lowering its performance at the same time.

The goal of signal processing of BCI aims at extracting useful features from the data and translating them into command signal for further applications. The common used detection methods for SSVEP-based BCIs are power spectral density analysis (PSDA) [9], canonical correlation analysis (CCA) $[8,10]$, and signal-to-noise ratio (SNR) [11]. Because all these methods have different properties under different conditions, adjustment and testing are required before the adoption of these methods.

Researchers have used EEG for control of cursors, robotic arms, spellers, prostheses, wheelchairs, and other devices and try to move BCI systems out of the laboratory into patients' homes [12-14]. Particularly, having food is a basic requirement in the daily life. But there is few proposed BCI home health care systems that integrates the following features at the same time : non-invasive, wireless, easy to use, and enabling users to independently have meals.

In this study, by integrating a SSVEP-based BCI and a robotic eating assistive system, we proposed a wireless steady state visually evoked potential (SSVEP)-based BCI eating assistive system.

\section{MATERIAL AND METHODS}

\section{A. Introduction}

Figure 1A shows the configuration of the system. The SSVEP BCI system is composed of a monitor, a computer, and a wireless EEG cap, Mindo 4S (developed by Brain Research Center of National Chiao Tung University). The eating assistive system contains the robotic arm, Jaco, a meal box, and a web camera for mouth position detection. The speakers are for voice instruction. There are five options including three types of food, a bottle of water and an exit option on the menu. When subjects start the system, the voice instructions will get them relaxed, concentrated, and inform them how to select the intentional 
service. When subjects select and keep staring at a target function on the main menu, the system detects and processes the underlying EEG pattern in real time. The information is subsequently used to trigger the selected service system. Figure 1B shows the block diagram of the integrated eating assistive system. As shown, the SSVEP BCI system transforms user's EEG signal into commands and then triggers the selected services of eating assistive system, and Jaco will execute the task in accordance with the command and mouth position.

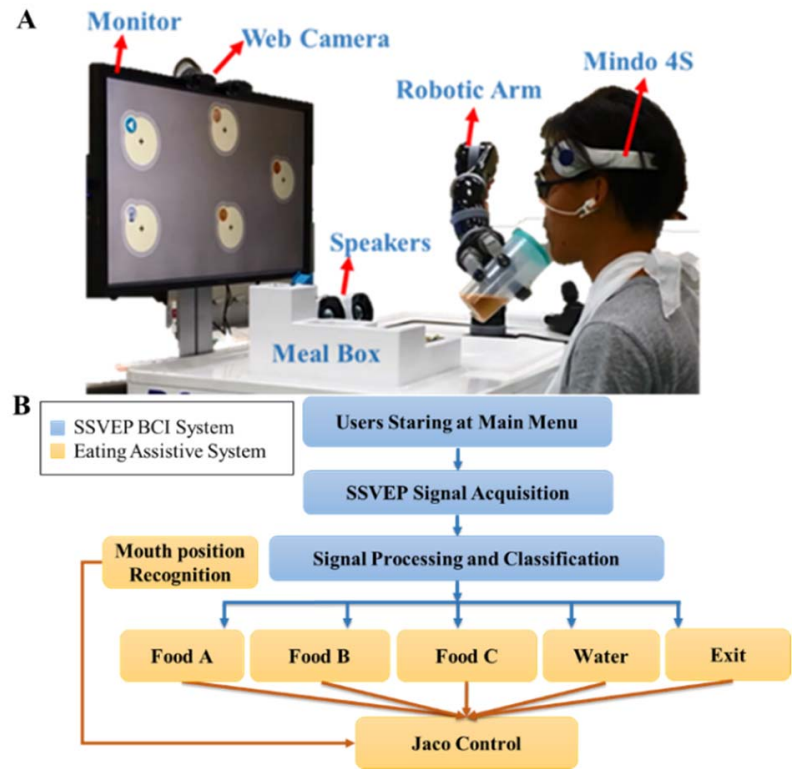

Figure 1. Wireless SSVEP-based BCI eating assistive system. (A) System configuration. The components of this system are shown here. The high refresh rate monitor and the wireless EEG cap, Mindo 4S are adopted for SSVEP stimulus presentation and signal acquisition. The robotic arm, meal box and the web camera for mouth position detection are components to realize eating assistance. (B) Block diagram of the integrated BCI eating assistive system. When the user stares at the menu, the SSVEP BCI system acquires and transforms user's EEG signal into commands via signal processing and classification, and then triggers the selected service of the eating assistive system. Once a service triggered, ex. Water, Jaco will deliver the selected food to the mouth position detected by mouth position recognition program. If an exit option is selected, Jaco will put back the spoon/bottle and go back to standby position.

\section{B. System Integration}

In this study, a wireless SSVEP-based BCI and a robotic eating assistive system are integrated in a platform developed in C\#. The details of each system are illustrated below.

The wireless SSVEP-based BCI is the core of this system. It judges the user's intention according to the recorded EEG data and sends the command to the corresponding subsystem to provide the requested service to the user. The advantage of wireless was realized a wireless EEG cap, Mindo 4S. Circleshaped visual stimuli (white and black, 100\% contrast, frequencies: $14.4 \mathrm{~Hz}, 16 \mathrm{~Hz}, 18 \mathrm{~Hz}, 20.6 \mathrm{~Hz}, 24 \mathrm{~Hz}$ ) were coded at a monitor with refresh rate of $144 \mathrm{~Hz}$. Experimental procedures were designed for optimization and evaluation of the system. The visual stimulus was presented by Unity (a cross-platform game-engine developed by Unity Technologies). The Details of the experiment will be shown after system integration.
The robotic eating assistive system provides the eating assistive function of this system. The dominating component here is the robotic arm, Jaco (AM 32400003 of Kinova). Through the application programming interface of Jaco, the SSVEP-based BCI can command Jaco to execute specific trajectories previously recorded. Due to the reason that every user has different mouth position, a mouth position detecting software and a webcam are integrated into this system to instruct Jaco where to stop.

\section{Experimental Setup}

A four-channel wireless, portable, lightweight EEG cap (Mindo 4S [15], developed by NCTU BRC) was used for EEG recording. In this study, two of the four channels were adopted with spring-loaded dry sensors, and the cap wearing position was adjusted to $\mathrm{O} 1, \mathrm{O} 2$ channels according to the 10-20 International Standard. With the special made spring-loaded sensors, EEG data with quality comparable to that obtained with wet-electrode systems was recorded without the need of skin preparation or conductive gels [16]. The EEG signals were then amplified, band-pass filtered $(0.24-125 \mathrm{~Hz})$, digitized (sampling rate: $250 \mathrm{~Hz}$ ).

For the BCI experiment, a high level stimulating monitor with $144 \mathrm{~Hz}$ refresh rate (BenQ XL2430T) was used to present the stimuli. Flickering frequencies of $14.4 \mathrm{~Hz}, 16 \mathrm{~Hz}, 18 \mathrm{~Hz}$, $20.6 \mathrm{~Hz}, 24 \mathrm{~Hz}$, which correspond to ten, nine, eight, seven, six frames in one flickering period are used in this research. The stimulation patterns of this system are shown in Figure 2A. The stimulus presentation follows the proposed method [17]. The layout of the menu is shown in Figure 2B.
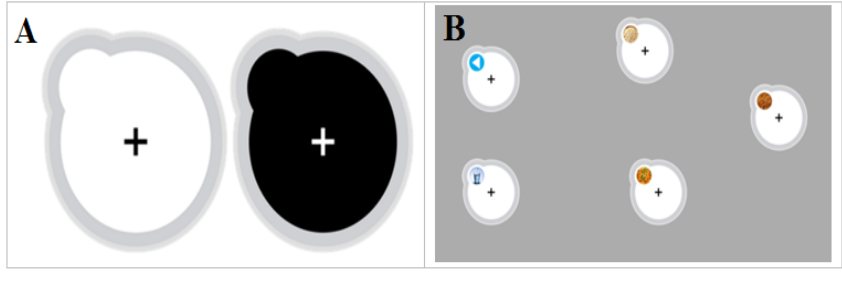

Figure 2 Layouts of the stimulus. (A) Stimulation pattern of this system. The flickering of this system is presented by switching of black and white patterns as shown here. (B) Menu of the system. The menu contains five options including three kinds of food, water, and an exit option.

In this study, the EEG data is collected from 15 healthy participants (thirteen males and two females and they are all right-handed adults (overall mean age $23 \pm 2.3$ years) with normal or corrected-to normal vision. They have no history of neurological or psychological disorders (such as migraine or epilepsy). They read and signed an informed consent form approved by the Research Ethics Committee for Human Research Protections. After a detailed explanation of the experimental procedure, all participants completed a consent form before the experiment. The subjects sat in a comfortable chair approximately $55 \mathrm{~cm}$ from the monitor, and gazed at one of the visual stimuli. The experiment consisted of five bouts, during which subjects had to gaze at a specific stimulus for ten seconds. The order of presented stimuli frequencies was from low to high. There were ten-second rest between two bouts. 


\section{Signal Analysis}

Each of the ten second recorded data for a specific frequency was converted into six five-second-long decisions (trials) as shown in Figure 3. Under this procedure, the system could make a decision in every five seconds using the five second data just recorded. Performances of data lengths from one second to seven seconds were tested before the adoption of five seconds, and the results in Figure 3 shows that five seconds would be the most efficient choice.

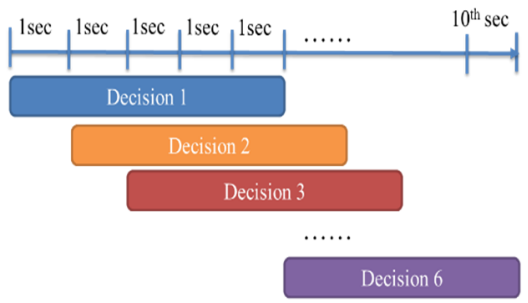

Figure 3. Schematic diagram of data segmentation. The first decision required the data from the zeroth second to the fifth second. Similarly, the second decision required the data from the first second to sixth second and so on. Following the same rule, the sixth decision required the fifth second to tenth second.

For each decision from each recorded channel, fast Fourier transform (FFT) was applied, and signal-to-noise ratio (SNR) at each single channel was afterward computed using equation (21), where $P_{n}$ is the power value of $\mathrm{n} \mathrm{Hz}$.

$$
\mathrm{SNR}\left(\text { of n Hz) }=\frac{P_{n}}{\left(P_{n-1}+P_{n-0.5}+P_{n+0.5}+P_{n+1}\right) / 4}\right.
$$

As Canonical-correlation analysis (CCA) has been frequently used in SSVEP-based BCI and proved to be efficient for frequency detection, we adopted it in this study and compare it with another method we developed. For two given data sets $X=$ $\left(x_{1}, x_{2}, \ldots, x_{p}\right)$ and $Y=\left(y_{1}, y_{2}, \ldots, y_{q}\right)$, what CCA does is to find a pair of linear transforms $\left(W_{x}, W_{y}\right)$ for $\mathrm{X}$ and $\mathrm{Y}$ such that the correlation between the two canonical variates $x^{*}$ and $y^{*}$ are maximized according to the equation (2-2).

$$
\begin{aligned}
& \max _{W_{x}, W_{y}} \rho\left(x^{*}, y^{*}\right)= \\
& \frac{E\left[W_{x}^{T} X Y^{T} W_{y}\right]}{\sqrt{E\left[W_{x}^{T} X X^{T} W_{x}\right] E\left[W_{y}^{T} Y Y^{T} W_{y}\right]}}=\frac{W_{x}^{T} C_{x y} W_{y}}{\sqrt{W_{x}^{T} C_{x x} W_{x} W_{y}^{T} C_{y y} W_{y}}} \\
& x^{*} \text { and } y^{*} \text { can be computed as equation (2-3) and } \\
& x^{*}=x_{1}^{T} w_{x_{1}}+\cdots+x_{p}^{T} w_{x_{p}}=X^{T} W_{x} \\
& y^{*}=y_{1}^{T} w_{y_{1}}+\cdots+y_{q}^{T} w_{y_{q}}=Y^{T} W_{y}
\end{aligned}
$$$$
x^{*} \text { and } y^{*} \text { can be computed as equation (2-3) and (2-4). }
$$

In this study, $\mathrm{X}$ is the EEG data and $\mathrm{Y}$ is the reference signal formulated according to equation (2-5) where $f_{1}, f_{2}, \ldots, f_{k}$ are the frequencies of stimulations, and $\mathrm{n}$ is the length of the EEG signal, and $\mathrm{N}$ is the number of sampling points and $f_{s}$ is the sampling rate. We use the EEG signal from $\mathrm{O} 1$ and $\mathrm{O} 2$ channels and reference signal as input for the CCA method. CCA calculates the canonical correlation $\rho$ between the EEG data and reference signals. The frequency of the reference signal that produces maximal correlation was more likely to be the selected target or the harmonic of the selected target.
Reference data $: Y=\left[\begin{array}{c}\sin \left(2 \pi f_{1} n\right) \\ \cos \left(2 \pi f_{1} n\right) \\ \sin \left(2 \pi f_{2} n\right) \\ \cos \left(2 \pi f_{2} n\right) \\ \vdots \\ \sin \left(2 \pi f_{k} n\right) \\ \cos \left(2 \pi f_{k} n\right)\end{array}\right], n=\frac{1}{f_{s}}, \frac{2}{f_{s}}, \frac{3}{f_{s}}, \ldots, \frac{N}{f_{s}}$

Due to the fact that this system was built into multilayer structure and contains an option of switching function page in every menu, misjudgement of classification may waste users lots of time to switch back. Therefore, a voting method was developed to allow the system to skip trials without clear intention to reduce error rate. Figure 4 shows the structure of the voting method. For each channel's data both FFT and SNR results can provide one answer. If there were three or more answers vote for a specific option, the algorithm would choose that option. If not, the algorithm would skip the trial and wait until the threshold was reached.

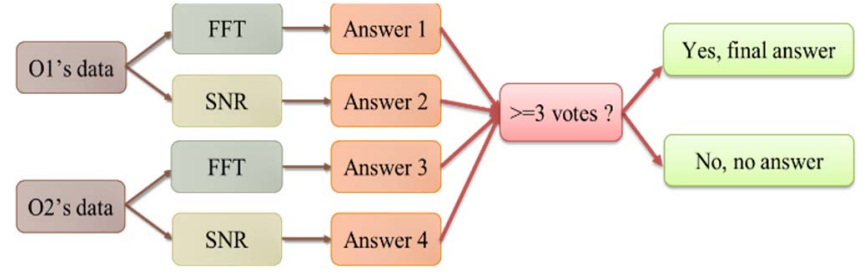

Figure 4. Structure of four vote mechanism. FFT and SNR are calculated from each channel's data and then produce four answers. A final answer will be made if three or more answers vote for one specific option. Otherwise, the system will skip this trial and move on to next one.

\section{RESULTS AND DisCUSSION}

To evaluate the performance of this system, Information transfer rate (ITR) and accuracy of the recorded data were calculated. Accuracies of different classification methods were also compared to optimize the system. Besides, an ALS patient was invited for the online performance evaluation and to provide suggestions for future improvement. Considering there may be skipped trials in voting method, calculation of accuracy is defined as the ratio of the number of corrected decisions and the number of answered ones.

\section{A. Accuracies and ITR}

Figure 5 shows classification accuracies using different length of recorded data. As shown, the accuracy exceeds $90 \%$ when data length reaches five seconds, and the performance of five, six, and seven seconds are quite close. Therefore, five-second length is adopted in this system.

Table 3-1 shows the results of the offline data. Each row of the table shows the accuracy, number of correct or wrong trials, and ITR of the offline data corresponding to the applied method. As shown, before the adoption of voting method, the accuracies of all the other methods were all below 90\% and with large variance. Since the number of adopted channels affects the performance of CCA, the accuracies of CCA was not ideal in this two channel system. Because the voting method skipped the trials without clear features/intentions of the subjects. The trials which are more likely to be misclassified are skipped. Therefore, accuracies are significantly improved. Although the number of correct trials was suppressed, the amount of wrong trials was 
dramatically suppressed, too. Therefore, the ITR performance was improved. In general, the adoption of voting method improves the performance of the system, and provides less incorrect trials, which makes the system more applicable.

\section{B. User feedback}

User feedback of this system was collected via questionnaire and verbal communication with the invited ALS patient. Since the brain activities of patients are usually different from normal people, the results of ALS patient is separated from healthy subjects'. The patients recorded data achieved accuracy of $73.9 \%$ and ITR of 11.67 (bit per min) which are lower than averaged results of healthy subjects. Basically, the patient agreed that the operating procedure was convenient, the provided functions met their need, the movement of the robotic arm was safe, and there wasn't any discomfort when wearing wireless dry sensor EEG cap or staring at the flickering. And some suggestions were also provided. Since people with spinal disabilities or other severe disabilities may have problem moving their body forward, even a little bit, it's hard for these users to eat the food in front of them. Therefore, it's suggested that we adjust the movement design of the robotic arm to deliver the food to mouth at appropriate moments instead of waiting at mouth position for users to get it. On the other hand, the usage of SSVEP BCI control requires much attention. The patient felt it wasn't easy to concentrate for a long time throughout the process. Therefore, improvement of the sensibility and accuracy of the system is necessary.

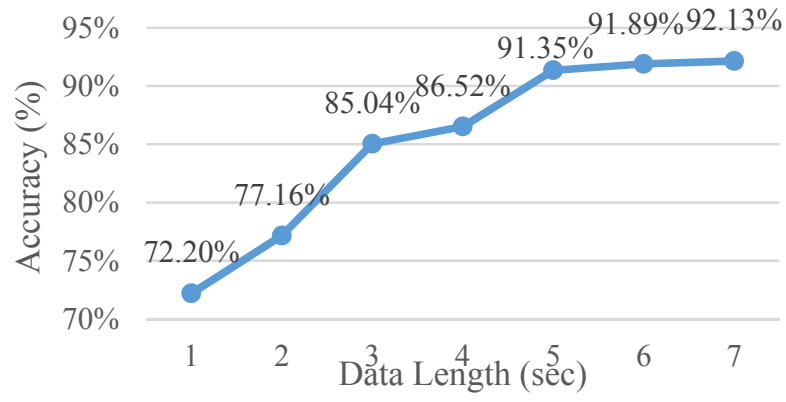

Figure 5. Classification accuracy using voting method with different data lengths. The accuracies become quite close when using five, six or seven seconds as length of data.

TABLE 3-1, PERFORMANCE OF OFFLINE DATA. TOTAL NUMBER OF TRIALS: 450.

\begin{tabular}{|c|c|c|c|c|c|}
\hline Method & Data & ACC & $\begin{array}{c}\text { \# of correct } \\
\text { trials }\end{array}$ & $\begin{array}{c}\text { \# of wrong } \\
\text { trials }\end{array}$ & ITR \\
\hline FFT & o1 & $55.78 \%$ & 251 & 199 & 5.37 \\
\hline FFT & o2 & $69.78 \%$ & 314 & 136 & 10.00 \\
\hline SNR & o1 & $54.22 \%$ & 244 & 206 & 4.94 \\
\hline SNR & o2 & $76.00 \%$ & 342 & 108 & 12.56 \\
\hline CCA & o1, o2 & $80.22 \%$ & 361 & 89 & 14.51 \\
\hline 4 Votes & o1, o2 & $91.35 \%$ & 243 & 23 & 20.69 \\
\hline
\end{tabular}

\section{CONCLUSIONS}

In this study, we proposed a non-invasive wireless SSVEPbased BCI eating assistive system which enables users to have meals independently and it also has great potential for improving user experience of homecare systems and reducing burden of caregivers. For this brand new system which adopted only two channels, we also developed classification algorithms to improve the accuracy and lower the amount of wrong trials. The average accuracy of this system achieved $91.4 \%$.

However, this system still has disadvantages to be improved. According to the invited Amyotrophic Lateral Sclerosis patient, the design of movement of the robotic arm should be adjust to deliver the food to users' mouth timely instead of waiting in a specific position. And the detection and classification of the system should also be more sensible because it is not easy for users to concentrate for a long time.

For future work, our group will mainly focus on improving the classification methods for there are still many features and combinations to be tested. Also, further investigations of different users' EEG characteristics are also important. Building models for different groups of users and optimize the parameters of the system based on the models during training time for the current user should theoretically improve the performance of the system.

\section{ACKNOWLEDGMENT}

This work was supported in part by the Aiming for the Top University Plan of National Chiao Tung University, sponsored by the Ministry of Education, Taiwan, under Grant Number 105W963; in part by the Cognition and Neuroergonomics Collaborative Technology Alliance Annual Program Plan, sponsored by the Army Research Laboratory under Cooperative Agreement Number W911NF-10-2-0022; in part by the VGHUST Joint Research Program, Tsou's Foundation, Taiwan, under Contract VGHUST105-G7-10-3. The authors would also like to thank Foxconn technology group, Taiwan.

\section{REFERENCES}

[1] A. H. Do, P. T. Wang, C. E. King, A. Abiri, and Z. Nenadic, "BrainComputer Interface Controlled Functional Electrical Stimulation System for Ankle Movement," Journal of NeuroEngineering and Rehabilitation, vol. 8, p. 49, 2011.

[2] B. Graimann, B. Allison, and G. Pfurtscheller, "Brain-Computer Interfaces: A Gentle Introduction," in Brain-Computer Interfaces, B. Graimann, G. Pfurtscheller, and B. Allison, Eds. Springer Berlin Heidelberg, 2009, pp. 1-27.

[3] P. S. Hammon and V. R. de Sa, "Preprocessing and meta-classification for brain-computer interfaces," IEEE Trans Biomed Eng, vol. 54, no. 3, pp. 518-525, Mar. 2007.

[4] A. Nijholt et al., "Brain-Computer Interfacing for Intelligent Systems," IEEE Intelligent Systems, vol. 23, no. 3, pp. 72-79, May 2008.

[5] C. T. Lin et al., "Review of Wireless and Wearable Electroencephalogram Systems and Brain-Computer Interfaces - A Mini-Review," Gerontology, vol. 56, no. 1, pp. 112-119, 2010.

[6] R. Fazel-Rezai, B. Z. Allison, C. Guger, E. W. Sellers, S. C. Kleih, and A. Kübler, "P300 brain computer interface: current challenges and emerging trends," Front Neuroeng, vol. 5, Jul. 2012.

[7] D. Regan, "Steady-state evoked potentials," J. Opt. Soc. Am., JOSA, vol. 67, no. 11, pp. 1475-1489, Nov. 1977.

[8] Y. P. Lin, Y. Wang, and T. P. Jung, “A mobile SSVEP-based braincomputer interface for freely moving humans: the robustness of canonical correlation analysis to motion artifacts," Conf Proc IEEE Eng Med Biol Soc, vol. 2013, pp. 1350-1353, 2013.

[9] G. Hakvoort, B. Reuderink, and M. Obbink, "Comparison of PSDA and CCA detection methods in a SSVEP-based BCI-system," Centre for 
Telematics and Information Technology, University of Twente, Enschede, info:eu-repo/semantics/report TR-CTI, Feb. 2011.

[10] Y.T. Wang, M. Nakanishi, Y. Wang, C.S. Wei, C.K. Cheng, and T.P. Jung, "An Online Brain-Computer Interface Based on SSVEPs Measured From Non-Hair-Bearing Areas," IEEE Trans Neural Syst Rehabil Eng, vol. 25, no. 1, pp. 11-18, Jan. 2017.

[11] P. Riyahi and A. Eskandarian, "Analyzing Steady-State Visual Evoked Potentials for Effective User Response Detection for Brain-Computer Interfaces," p. V03AT03A081, Nov. 2013.

[12] T. M. Vaughan et al., "The Wadsworth BCI Research and Development Program: at home with BCI," IEEE Trans Neural Syst Rehabil Eng, vol. 14, no. 2, pp. 229-233, Jun. 2006.

[13] C. Neuper, G. R. Müller, A. Kübler, N. Birbaumer, and G. Pfurtscheller, "Clinical application of an EEG-based brain-computer interface: a case study in a patient with severe motor impairment," Clinical Neurophysiology, vol. 114, no. 3, pp. 399-409, Mar. 2003.

[14] H. Rw, H. J, and P. P, "Cerebral location of international 10-20 system electrode placement.," Electroencephalogr Clin Neurophysiol, vol. 66, no. 4, pp. 376-382, Apr. 1987.

[15] Y. T. Liu, C. T. Lin, C. H. Chuang, Y. K. Wang, S. H. Huang, J. T. King, S. A. Chen, and S. W. Lu, "Novel Neurotechnology and Computational Intelligence Method Applied to EEG-based Brain-Computer Interfaces," accepted by IEEE Systems, Man, and Cybernetics Magazine, 2016.

[16] C. T. Lin, L. D. Liao, Y. H. Liu, I. J. Wang, B. S. Lin, and J. Y. Chang, "Novel Dry Polymer Foam Electrodes for Long-Term EEG Measurement," IEEE Transactions on Biomedical Engineering, vol. 58, no. 5, pp. 1200-1207, May 2011.

[17] M. Nakanishi, Y. Wang, Y. T. Wang, Y. Mitsukura, and T. P. Jung, "Generating visual flickers for eliciting robust steady-state visual evoked potentials at flexible frequencies using monitor refresh rate," PloS one, vol. 9, no. 6, Jun. 2014. 